\title{
Locality and Causality in Hidden Variables Models of Quantum Theory
}

\author{
Stefan Teufel, Karin Berndl, Detlef Dürr \\ Mathematisches Institut der Universität München, \\ Theresienstraße 39, 80333 München, Germany \\ Sheldon Goldstein \\ Department of Mathematics, Rutgers University, \\ New Brunswick, NJ 08903, USA \\ Nino Zanghì \\ Istituto di Fisica dell'Università di Genova, \\ Istituto Nazionale di Fisica Nucleare, Sezione di Genova, \\ Via Dodecaneso 33, 16146 Genova, Italy
}

(April 30, 1997)

\begin{abstract}
Motivated by Popescu's example of hidden nonlocality, we elaborate on the conjecture that quantum states that are intuitively nonlocal, i.e., entangled, do not admit a local causal hidden variables model. We exhibit quantum states which either (i) are nontrivial counterexamples to this conjecture or (ii) possess a new kind of more deeply hidden irreducible nonlocality. Moreover, we propose a nonlocality complexity classification scheme suggested by the latter possibility. Furthermore, we show that Werner's (and similar) hidden variables models can be extended to an important class of generalized observables. Finally a result of Fine on the equivalence of stochastic and deterministic hidden variables is generalized to causal models.
\end{abstract}

03.65. Bz

Typeset using REVTEX 


\section{INTRODUCTION}

The nonlocality inherent in the quantum mechanical description of nature is still a much debated subject, 30 years after the pioneering work by John Bell [四]. Bell's starting point was the argument of Einstein, Podolsky, and Rosen (EPR) [2], demonstrating the existence of local hidden variables implying the incompleteness of the quantum mechanical description of a physical system by the wave function alone. The EPR argument is based on one essential assumption: locality, or local causality. Bell derived an inequality which the distributions of these local hidden variables have to satisfy and showed that the quantum mechanical values for these probabilities violate this inequality. Thus there cannot be a local hidden variables model for quantum theory. But Bell's argument in conjunction with the EPR argument actually shows much more: the quantum mechanical predictions cannot be explained by local physical laws. And since the quantum mechanical predictions are confirmed by most experiments, see for example [3], one has to conclude that there are nonlocal physical actions in nature.

EPR argued by considering a thought experiment with a certain correlated 2-particle state. Bohm transformed this thought experiment into one which is similar to actually performed experiments, involving spin measurements on the singlet state [4]. Does this EPR-Bell nonlocality arise only in such special situations, while most quantum systems are local? For most quantum states the EPR argument clearly does not apply since it requires perfect correlation between lots of pairs of results of widely separated measurements. Bell has introduced an alternative to the EPR-argument that applies to all quantum states: the concept of stochastic local hidden variables [1]. As has been noted (in a special case) by Fine [5], this concept actually leads to the same framework as the EPR argument, namely to (deterministic) local hidden variables.

Thus one may now ask which quantum states admit a local hidden variables model, i.e., are EPR-Bell-local. For pure quantum states one can rather easily see that for any entangled state of 2 or more particles one can find observables whose correlations violate a Bell inequality (see for example [6,7]). This implies that no local hidden variables model exists for such quantum states. Only pure product states $\psi=\psi^{(1)} \otimes \ldots \otimes \psi^{(N)}$ are EPRBell-local. Now what do we expect for mixed states? A mixed 2-particle state describing an ensemble of pure product states, i.e, a convex sum of product density matrices $\rho=$ $\sum_{\nu} p_{\nu} \rho_{\nu}^{(1)} \otimes \rho_{\nu}^{(2)}, p_{\nu}>0$, is also EPR-Bell-local. The question is whether any "entangled" density matrix, i.e., any density matrix that cannot be written as a mixture of product density matrices, is EPR-Bell-nonlocal. We certainly expect that such states exhibit nonlocal behavior because in any representation of the density matrix as a convex sum of pure states there are entangled wave functions present. The problem is how to reveal this nonlocality.

That this question is tricky is suggested by the existence of a class of density matrices discovered by Werner which are entangled but nevertheless admit a local hidden variables model 8 . The Werner states $W$ are states on the Hilbert space $\mathcal{H}=\mathbb{C}^{d} \otimes \mathbb{C}^{d}$ and may be thought of as describing two spin $(d-1) / 2$ particles:

$$
W=\frac{d+1}{d^{3}} I-\frac{1}{d^{2}} F,
$$

where $F$ is the flip operator, $F\left(\psi^{(1)} \otimes \psi^{(2)}\right)=\psi^{(2)} \otimes \psi^{(1)}$. Recently Popescu [9] pointed out an interesting property of Werner states. When we perform local measurements of the form 
$P=T \otimes I$ and $Q=I \otimes T$ on each subsystem, where $T$ is a projector on a two-dimensional subspace of $\mathbb{C}^{d}, T=|1\rangle\langle 1|+| 2\rangle\langle 2|$, we get nonlocal correlations in a sub-ensemble. The subensemble for which $P$ and $Q$ both yield the outcome 1, described by the collapsed density matrix

$$
W^{\prime}=\frac{(P Q) W(P Q)}{\operatorname{tr}(W P Q)}
$$

violates a Bell inequality for $d \geq 5$; thus there are local observables whose correlations in this new state can no longer be described by a local model. The nonlocality of $W$ intuitively corresponding to the property of $W$ of being entangled, but "hidden" when only a single measurement on each side is considered - can thus be revealed by sequences of local measurements.'

Since in Popescu's example the observables leading to a violation of a Bell inequality commute with $P$ and $Q$, these time sequences of measurements can be described by single observables, and Werner's model may be applied to it. However, the model thus obtained violates causality: later measurements influence preceding ones [9,11]. Since such a model does not appropriately represent the physical idea of locality - actions into the past are even worse than actions at a distance - we propose, following Popescu [9] and Mermin [11], as a requirement for a local hidden variables model that it satisfy the locality and causality conditions, defining an LCHV model, described here.2

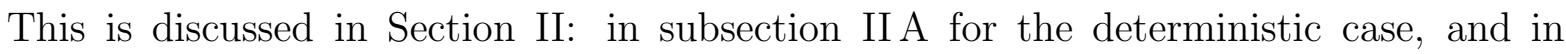
subsection IIB for the stochastic case. It turns out however that any quantum state that allows for a stochastic hidden variables model also allows for a deterministic model and vice versa. This is a generalization of a result of Fine (to causal models with arbitrary many observables).

In Section IIII we shall discuss our main conjecture about the classification of local states - that for $d>2$ no entangled density matrix on $\mathbb{C}^{d} \otimes \mathbb{C}^{d}$ admits an LCHV model. We can neither prove nor disprove this conjecture, but we shall discuss some partial results which shed light on it. For pure product states and mixtures of product states there exists, as one would certainly expect, an LCHV model. This is shown in Section IIIA. Likewise, for pure entangled states there can be no LCHV model. In Section IIIB we discuss Popescu's result in our terms: the Werner states (for $d \geq 5$ ) do not admit LCHV models. In Section IIIC we show that in $d=2$ dimensions the conjecture is false. All the same, the case $d=2$ is special, and the conjecture may well be correct for $d \geq 3$. We exhibit a larger class of states similar to the Werner states, which are entangled, admit a local hidden variables model, and have the additional property that after the first measurement on one side the projected states are mixtures of product states, i.e., local states. However, in contrast to the 2-dimensional case we cannot conclude that there is an LCHV model for these states.

\footnotetext{
${ }^{1}$ Gisin [10] has recently found entangled states in $d=2$ which do not violate certain types of Bell inequalities for single measurements but do so for sequences of (generalized) measurements.

${ }^{2}$ Recently, the causality condition for stochastic hidden variables models has also been discussed by Zukowski et al. [12].
} 
This situation is interesting: Either these states have an LCHV model and hence are counterexamples to our main conjecture, or they do not, in which case they possess a more deeply hidden irreducible nonlocality, different from that of the examples known so far, and suggesting a new nonlocality classification scheme, described in Section एIID.

In Section IV we shall consider generalized measurements: since Werner's local hidden variables model is a model for standard observables only, it may be possible, as has been conjectured by Popescu [9], to reveal the "hidden nonlocality" by generalized observables associated with positive operator valued (POV) measures. More generally, the existence of a local hidden variables model for generalized observables might be equivalent to the state being non-entangled. However, we show in Section $\mathbb{I V B}$ that local hidden variables models for standard observables can be extended to cover some POV measurements, which restricts the class of POV's that can be used to prove or disprove this conjecture.

\section{LOCAL CAUSAL HIDDEN VARIABLES MODELS}

\section{A. Deterministic hidden variables}

To motivate our definition of hidden variables models we shall briefly repeat the essentials of the EPR argument in Bohm's version. Consider two spin- $\frac{1}{2}$ particles in the singlet state. If the spin of each particle is measured in some direction a the two outcomes are perfectly anticorrelated, but the outcome of a particular measurement is completely random. If one particle has a-spin +1 the measurement of the a-spin of the other particle will yield -1 . The assumption of locality now implies that the particles carry the information about their spin value in every direction with them. To account for the perfect anticorrelation as well as for the randomness of the particular result, the adequate mathematical language is that of random variables $X_{\mathbf{a}}, X_{\mathbf{b}}$ for the spin in every direction $\mathbf{a}$ and $\mathbf{b}$, respectively, for both particles on a probability space $\Omega$ with a probability measure $\mathbb{P}$. We shall sometimes call this kind of hidden variables "deterministic hidden variables" - the hidden variable $\omega \in \Omega$ determines the results of all possible experiments - to distinguish them from the "stochastic

\footnotetext{
${ }^{3}$ The following analogy may be helpful. In classical statistical mechanics the microstate of a system is a point in phase space and the statistical properties of macroscopic systems are explained by considering macrostates, i.e., ensembles of systems in different microstates which are macroscopically indistinguishable. Observables are just random variables on phase space and their distributions are determined by the macrostate. Can the quantum mechanical observables be understood in a similar way? Systems with the same wave function would, in this analogy, belong to the same macrostate and the individual properties of a particular system would be determined by the microstate, a point in a yet unknown probability space, traditionally called the space of hidden variables. Quantum mechanical observables would be random variables on this new probability space and their distributions would agree with the quantum mechanical predictions for suitably chosen ensembles of microstates. (In particular, there would be joint distributions for all observables.)
} 
hidden variables" we shall introduce in the next subsection. Note, however, that determinism has been inferred from locality.

We shall consider the following general setup. The physical system is in a quantum state $\rho$, which is a density matrix on a Hilbert space $\mathcal{H}$. For the time being, we shall assume that only ideal (von Neumann) measurements are performed on the system, i.e., a measurement is associated with an observable, which is a self-adjoint operator on $\mathcal{H}$, and the usual collapse rule for sequences of ideal measurements applies. Observables are denoted by $O^{k}$, their spectral values by $o$, and their spectrum by $\sigma\left(O^{k}\right)$. Furthermore, we denote by $P_{o}^{k}$ the projection onto the eigenspace of the observable $O^{k}$ corresponding to the eigenvalue $o$. For simplicity we shall explicitly focus only on observables with discrete spectrum.

By a hidden variables model (HV model) for a quantum state $\rho$ we shall mean a probability space $(\Omega, \mathbb{P})$ and a family $X$ of random variables on $(\Omega, \mathbb{P})$ such that for any time-ordered sequence of (Heisenberg) observables $\left(O^{1}\left(t_{1}\right), \ldots, O^{n}\left(t_{n}\right)\right)$ with $t_{1}<\ldots<t_{n}$ of arbitrary length $n$ there is a corresponding random variable, a function

$$
X_{O^{1}\left(t_{1}\right), \ldots, O^{n}\left(t_{n}\right)}: \Omega \rightarrow \sigma\left(O^{1}\right) \times \ldots \times \sigma\left(O^{n}\right) \subset \mathbb{R}^{n},
$$

whose distribution agrees with the quantum mechanical distribution for the results of a sequence of ideal measurements of the observables $O^{1}\left(t_{1}\right), \ldots, O^{n}\left(t_{n}\right)$ :

$$
\begin{aligned}
& \mathbb{P}\left(X_{O^{1}\left(t_{1}\right), \ldots, O^{n}\left(t_{n}\right)}=\left(o_{1}, \ldots, o_{n}\right)\right) \\
& \quad=\operatorname{tr}\left(P_{o_{n}}^{n}\left(t_{n}\right) \cdots P_{o_{1}}^{1}\left(t_{1}\right) \rho P_{o_{1}}^{1}\left(t_{1}\right) \cdots P_{o_{n}}^{n}\left(t_{n}\right)\right) .
\end{aligned}
$$

This condition guarantees that the HV model captures all the quantum mechanical predictions for arbitrary (ideal) measurements in the state $\rho$. (If in the sequence $\left(O^{1}\left(t_{1}\right), \ldots, O^{n}\left(t_{n}\right)\right)$ some consecutive observables are jointly measurable, i.e., commute, we may allow the times of the consecutive commuting observables to be equal.)

For any quantum state $\rho$, there is a $\mathrm{HV}$ model: for example, take $(\Omega, \mathbb{P})$ to be the interval $[0,1]$ with uniform distribution (=Lebesgue measure), and define the random variables by splitting $[0,1]$ into pieces with lengths equal to the probabilities of the occurring values. Note that this trivial construction does not contradict the no-hidden-variables theorems. The proofs that there can be no hidden variables require additional properties of the hidden variables. One such property is that of "non-contextuality;" see [1, 13] for a discussion of this property and its limited physical relevance. We shall now consider physically relevant additional properties, namely locality and causality.

We split the random variable $X_{O^{1}\left(t_{1}\right), \ldots, O^{n}\left(t_{n}\right)}$ that describes a sequence of $n$ measurements into an initial segment $X_{O^{1}\left(t_{1}\right), \ldots, O^{n-1}\left(t_{n-1}\right)}^{O^{n}\left(t_{n}\right.}$ of length $n-1$ describing the outcome of the first $n-1$ measurements which in general depends on the later measurement $O^{n}\left(t_{n}\right)$, and a final ("follow-up") random variable $X_{O^{n}\left(t_{n}\right)}^{O^{1}\left(t_{1}\right), \ldots, O^{n-1}\left(t_{n-1}\right)}$, describing the outcome of the last experiment $O^{n}\left(t_{n}\right)$ depending on the first $n-1$ measurements:

$$
\begin{aligned}
& X_{O^{1}\left(t_{1}\right), \ldots, O^{n}\left(t_{n}\right)} \\
& \quad=:\left(X_{O^{1}\left(t_{1}\right), \ldots, O^{n-1}\left(t_{n-1}\right)}^{O^{n}\left(t_{n}\right)}, X_{O^{n}\left(t_{n}\right)}^{O^{1}\left(t_{1}\right), \ldots, O^{n-1}\left(t_{n-1}\right)}\right) .
\end{aligned}
$$

A causal hidden variables model ( $\mathrm{CHV}$ model) for a quantum state $\rho$ is a $\mathrm{HV}$ model satisfying the causality condition that initial segments do not depend upon what measurements are later performed, i.e., that 


$$
X_{O^{1}\left(t_{1}\right), \ldots, O^{n-1}\left(t_{n-1}\right)}^{O^{n}\left(t_{1}\right)}=X_{O^{1}\left(t_{1}\right), \ldots, O^{n-1}\left(t_{n-1}\right)} .
$$

Equivalently, the HV model is causal if

$$
\begin{aligned}
& X_{O^{1}\left(t_{1}\right), \ldots, O^{n}\left(t_{n}\right)} \\
& \quad=\left(X_{O^{1}\left(t_{1}\right)}, X_{O^{2}\left(t_{2}\right)}^{O^{1}\left(t_{1}\right)}, \ldots, X_{O^{n}\left(t_{n}\right)}^{O^{1}\left(t_{1}\right), \ldots, O^{n-1}\left(t_{n-1}\right)}\right) .
\end{aligned}
$$

This condition captures the physical idea of causality: the description of an experiment associated with $O^{m}\left(t_{m}\right)$ may depend on those experiments performed earlier, but it is not changed by experiments that are performed in the future. If, for example, two sequences of observables are considered differing in the later observable, $\left(O^{1}\left(t_{1}\right), O^{2}\left(t_{2}\right)\right), t_{1}<t_{2}$, and $\left(O^{1}\left(t_{1}\right), O^{2^{\prime}}\left(t_{2}^{\prime}\right)\right), t_{1}<t_{2}^{\prime}$, then

$$
X_{O^{1}\left(t_{1}\right), O^{2}\left(t_{2}\right)}=\left(X_{O^{1}\left(t_{1}\right)}, X_{O^{2}\left(t_{2}\right)}^{O^{1}\left(t_{1}\right)}\right)
$$

and

$$
X_{O^{1}\left(t_{1}\right), O^{2}\left(t_{2}^{\prime}\right)}=\left(X_{O^{1}\left(t_{1}\right)}, X_{O^{2^{\prime}}\left(t_{2}^{\prime}\right)}^{O^{1}\left(t_{1}\right)}\right),
$$

and the outcome of $O^{1}\left(t_{1}\right)$ does not depend upon which, if any, measurements are performed later.

Just as for HV models, one can readily see, since the quantum probabilities ([3) are, in an obvious sense, causal, that all quantum mechanical states admit a CHV model. Take $(\Omega, \mathbb{P})$ to be $[0,1]$ with uniform distribution, define the one-observable random variables $X_{O^{1}\left(t_{1}\right)}$ for all $O^{1}\left(t_{1}\right)$ as explained above, and define then inductively on each of the subsets $\left\{X_{O^{1}\left(t_{1}\right), \ldots, O^{n-1}\left(t_{n-1}\right)}=\left(o_{1}, \ldots, o_{n-1}\right)\right\}$ the follow-up random variables $X_{O^{n}\left(t_{n}\right)}^{O^{1}\left(t_{1}\right) \ldots, O^{n-1}\left(t_{n-1}\right)}$ by further splitting according to the conditional probabilities of the occurring values [14].

To discuss locality, we consider the following generalized EPR situation. The system is in a quantum state $\rho$ on the Hilbert space $\mathcal{H}=\mathcal{H}^{(1)} \otimes \mathcal{H}^{(2)}$, the factors of which correspond to two subsystems that are spatially separated such that no signal traveling at most at the speed of light will be able to propagate between them while two observers are locally conducting their experiments. Local measurements are described by observables of the form $A=A^{(1)} \otimes I$, on system 1 , and $B=I \otimes B^{(2)}$, on system 2. As a matter of convenience, when considering a sequence of local measurements we shall rearrange the random variables describing the successive measurements, placing those for system 2 to the right of those for system 1 and writing $X_{A^{1}, \ldots, A^{n}, B^{1}, \ldots, B^{m}}$ accordingly, regardless of their relative time ordering.f (Note that since in this setup all the $A$ 's commute with all the $B$ 's, we may collect together the $A$ 's and the $B$ 's in formula (3) as well.)

A local hidden variables model (LHV model) is a HV model satisfying the locality condition that the random variables describing the outcomes of local measurements on one system do not depend on the measurements performed on the other system, i.e.,

$$
X_{A^{1}, \ldots, A^{n}, B^{1}, \ldots, B^{m}}=\left(X_{A^{1}, \ldots, A^{n}}, X_{B^{1}, \ldots, B^{m}}\right) .
$$

\footnotetext{
${ }^{4}$ To simplify the notation we will sometimes drop the time variable of the Heisenberg operators.
} 
This condition says that while the results of local measurements in the two separated subsystems may be correlated, as for example are the colors of Bertlmann's socks [1], the local measurements performed on one system do not themselves influence those on the other system.

For the case of one local observable for each subsystem, this condition reduces to the condition that we have random variables $X_{A}$ and $X_{B}$ for all observables $A$ and $B$ for each subsystem - so that $X_{A, B}=\left(X_{A}, X_{B}\right)$ - such that the pair distributions agree with the quantum mechanical ones. This is the traditional framework of local hidden variables. The causality condition is obsolete in this case. We shall abbreviate by LHV1 model such an LHV model for single measurements, which we shall often speak of as measurements at a single time.

Finally we define a local causal hidden variables model (LCHV model) as a HV model that is both local and causal; and an LCHVk model as a model for sequences of measurements of length $k$ on both sides. (LCHV1 = LHV1.)

\section{B. Stochastic hidden variables}

Bell has introduced also so-called "stochastic local hidden variables models," where the hidden variable doesn't determine the measurement results completely, so that given the hidden variable the results are still random. The role of the hidden variables is to $e x$ plain correlations between measurement results at distant places: Assuming locality, it must be possible to identify sufficiently many causal factors=hidden variables, such that the residual fluctuations of the outcomes of experiments will be independent if these causal factors=hidden variables are held fixed (see Bell [1], pp. 150).

A stochastic local causal HV model for a quantum state $\rho$ consists of a probability space $(\widetilde{\Omega}, \widetilde{\mathbb{P}})$ and a family of maps $Q_{O^{1}\left(t_{1}\right), \ldots, O^{n}\left(t_{n}\right)}$ : for any time-ordered sequence of arbitrary length $n$ of observables $\left(O^{1}\left(t_{1}\right), \ldots, O^{n}\left(t_{n}\right)\right)$ with $t_{1}<\ldots<t_{n}$ there is a map from $\widetilde{\Omega}$ into probability distributions on the product of the spectra $\sigma\left(O^{1}\right) \times \ldots \times \sigma\left(O^{n}\right)$ denoted by

$$
Q_{O^{1}\left(t_{1}\right), \ldots, O^{n}\left(t_{n}\right)}: \widetilde{\omega} \mapsto Q_{O^{1}\left(t_{1}\right), \ldots, O^{n}\left(t_{n}\right)}(\cdot, \ldots, \cdot \mid \widetilde{\omega})
$$

with the following properties: (a) By averaging over $\widetilde{\Omega}$ the quantum mechanical probabilities are obtained

$$
\begin{aligned}
& \int_{\widetilde{\Omega}} Q_{O^{1}\left(t_{1}\right), \ldots, O^{n}\left(t_{n}\right)}\left(o_{1}, \ldots, o_{n} \mid \widetilde{\omega}\right) d \widetilde{\mathbb{P}}(\widetilde{\omega}) \\
& \quad=\operatorname{tr}\left(P_{o_{n}}^{n}\left(t_{n}\right) \cdots P_{o_{1}}^{1}\left(t_{1}\right) \rho P_{o_{1}}^{1}\left(t_{1}\right) \cdots P_{o_{n}}^{n}\left(t_{n}\right)\right) .
\end{aligned}
$$

(b) Causality: The marginals do not depend on later measurements

$$
\begin{aligned}
& Q_{O^{1}\left(t_{1}\right), \ldots, O^{n}\left(t_{n}\right)}\left(o_{1}, \ldots, o_{n-1} \mid \widetilde{\omega}\right) \\
& \quad \equiv \sum_{o_{n} \in \sigma\left(O^{n}\right)} Q_{O^{1}\left(t_{1}\right), \ldots, O^{n}\left(t_{n}\right)}\left(o_{1}, \ldots, o_{n-1}, o_{n} \mid \widetilde{\omega}\right) \\
& \quad=Q_{O^{1}\left(t_{1}\right), \ldots, O^{n-1}\left(t_{n-1}\right)}\left(o_{1}, \ldots, o_{n-1} \mid \widetilde{\omega}\right)
\end{aligned}
$$

equivalently 


$$
\begin{aligned}
Q_{O^{1}\left(t_{1}\right), \ldots, O^{n}\left(t_{n}\right)}\left(o_{1}, \ldots, o_{n} \mid \widetilde{\omega}\right) \\
=Q_{O^{1}\left(t_{1}\right), \ldots, O^{n-1}\left(t_{n-1}\right)}\left(o_{1}, \ldots, o_{n-1} \mid \widetilde{\omega}\right) \\
\quad \cdot Q_{O^{1}\left(t_{1}\right), \ldots, O^{n}\left(t_{n}\right)}\left(o_{n} \mid o_{1}, \ldots, o_{n-1}, \widetilde{\omega}\right) \\
=Q_{O^{1}\left(t_{1}\right)}\left(o_{1} \mid \widetilde{\omega}\right) Q_{O^{1}\left(t_{1}\right), O^{2}\left(t_{2}\right)}\left(o_{2} \mid o_{1}, \widetilde{\omega}\right) \\
\\
\quad \cdots Q_{O^{1}\left(t_{1}\right), \ldots, O^{n}\left(t_{n}\right)}\left(o_{n} \mid o_{1}, \ldots, o_{n-1}, \widetilde{\omega}\right)
\end{aligned}
$$

with the conditional probabilities

$$
\begin{aligned}
& Q_{O^{1}\left(t_{1}\right), \ldots, O^{n}\left(t_{n}\right)}\left(o_{k+1}, \ldots, o_{n} \mid o_{1}, \ldots, o_{k}, \widetilde{\omega}\right) \\
& =\frac{Q_{O^{1}\left(t_{1}\right), \ldots, O^{n}\left(t_{n}\right)}\left(o_{1}, \ldots, o_{k}, o_{k+1}, \ldots, o_{n} \mid \widetilde{\omega}\right)}{Q_{O^{1}\left(t_{1}\right), \ldots, O^{k}\left(t_{k}\right)}\left(o_{1}, \ldots, o_{k} \mid \widetilde{\omega}\right)} .
\end{aligned}
$$

(c) Locality: In the same sort of EPR framework as described earlier, the separated systems are conditionally independent given $\widetilde{\omega}$, i.e., for local measurements the probabilities $Q \ldots(\ldots \mid \widetilde{\omega})$ for fixed $\widetilde{\omega}$ factorize,

$$
\begin{aligned}
& Q_{A^{1}, \ldots, A^{n}, B^{1}, \ldots, B^{m}}\left(a_{1}, \ldots, a_{n}, b_{1}, \ldots, b_{m} \mid \widetilde{\omega}\right) \\
& \quad=Q_{A^{1}, \ldots, A^{n}}\left(a_{1}, \ldots, a_{n} \mid \widetilde{\omega}\right) Q_{B^{1}, \ldots, B^{m}}\left(b_{1}, \ldots, b_{m} \mid \widetilde{\omega}\right),
\end{aligned}
$$

where we have used the same rearrangement as earlier. Equivalently,

$$
\begin{gathered}
Q_{A^{1}, \ldots, A^{n}, B^{1}, \ldots, B^{m}}\left(a_{1}, \ldots, a_{n} \mid b_{1}, \ldots, b_{m}, \widetilde{\omega}\right) \\
=Q_{A^{1}, \ldots, A^{n}, B^{1}, \ldots, B^{m}}\left(a_{1}, \ldots, a_{n} \mid \widetilde{\omega}\right) \\
=Q_{A^{1}, \ldots, A^{n}}\left(a_{1}, \ldots, a_{n} \mid \widetilde{\omega}\right)
\end{gathered}
$$

and analogously for the other system. (The first equality directly above is usually called "outcome independence" and the second one "parameter independence.") In the same spirit we may define stochastic HV, CHV, LHV1, LCHV, and LCHVk models.

The existence of a deterministic model for a quantum state trivially implies the existence of a stochastic model with the same locality and causality properties. Interestingly, the converse is also true: From a stochastic model for a quantum state one may construct a deterministic model with the same locality and causality properties. Fine has proven the equivalence between the existence of deterministic and stochastic LHV1 models [5]. While he explicitly considers models involving only 4 observables, the basic idea of his proof extends to an arbitrary number, and in fact to all LHV models as well. We show in Appendix A that the equivalence between the existence of deterministic and stochastic models holds also for LCHV models.

This result is interesting for several reasons. Firstly, it reduces the complexity of a classification of states concerning nonlocality, since any state allowing for a stochastic model of a certain kind automatically also allows for a deterministic model with the same properties and vice versa. Secondly, when proving the existence of a HV model for a certain quantum state, we may use Werner's construction of a stochastic HV model and conclude that there is also a deterministic model. We shall use similar reasoning in the following discussion several times. Thirdly, we wish to remark that the equivalence between the existence of deterministic and stochastic HV models is also conceptually interesting with respect to 
the discussion of physical nonlocality. The EPR argument shows that locality implies the existence of deterministic LHV, while Bell's argument, as explained at the beginning of Section [1B, derives the concept of stochastic LHV from locality. These two seemingly different approaches are actually equivalent.

\section{IS THE NONEXISTENCE OF AN LCHV MODEL EQUIVALENT TO THE STATE BEING ENTANGLED?}

\section{A. The case of pure states and non-entangled density matrices}

Pure entangled states do not admit a local hidden variables model [1,66,7]. Thus there certainly can be no LCHV model for such states.

For quantum states which are a mixture of product density matrices one can construct an LCHV model by appropriately "mixing" the LCHV models for the individual terms. For completeness, we briefly give the natural construction. Consider for simplicity a finite sum $\rho=\sum_{\nu=1}^{n} p_{\nu} \rho_{\nu}^{(1)} \otimes \rho_{\nu}^{(2)}$ with $p_{\nu} \geq 0$ for all $\nu, \sum_{\nu=1}^{n} p_{\nu}=1$, and $\left(\Omega^{(1)}, \mathbb{P}^{(1)}, X^{(1, \nu)}\right)$, $\left(\Omega^{(2)}, \mathbb{P}^{(2)}, X^{(2, \nu)}\right)$ CHV models for $\rho_{\nu}^{(1)}$ and $\rho_{\nu}^{(2)}$. (For all $\nu$, the probability space $\left(\Omega^{(i)}, \mathbb{P}^{(i)}\right)$ may be chosen to be $[0,1]$ with Lebesgue measure.) Define $\Omega:=\Omega^{(1)} \times \Omega^{(2)} \times\{1, \ldots, n\}$ and $\mathbb{P}:=\mathbb{P}^{(1)} \times \mathbb{P}^{(2)} \times p$, where $p(\{\nu\})=p_{\nu}$ for $\nu \in\{1, \ldots, n\}$. For sequences of local observables $A^{i}=A^{(1) i} \otimes I, B^{j}=I \otimes B^{(2) j}$ let

$$
\begin{aligned}
& X_{A^{1}, \ldots, A^{n}, B^{1}, \ldots, B^{m}}(\omega) \\
& \quad:=\left(X_{A^{(1) 1}, \ldots, A^{(1) n}}^{(1, \nu)}\left(\omega^{(1)}\right), X_{B^{(2) 1}, \ldots, B^{(2) m}}^{(2, \nu)}\left(\omega^{(2)}\right)\right),
\end{aligned}
$$

$\omega=\left(\omega^{(1)}, \omega^{(2)}, \nu\right)$. One readily sees that this is an LCHV model for $\rho$. Thus all nonentangled density matrices are local in the sense that they admit an LCHV model. (This of course covers non-entangled pure states.)

By a construction similar to the one just given, one sees that the set of states (in some fixed Hilbert space) admitting an LCHV model, like the set of non-entangled states, is convex, i.e., if $\rho_{1}$ and $\rho_{2}$ admit an LCHV model, then so does $t \rho_{1}+(1-t) \rho_{2}$ for $0<t<1$.

In section $\mathrm{C}$ below we exhibit for $d=2$ an entangled density matrix that nevertheless admits an LCHV model. However, as will be seen in section $\mathrm{C}$, the case $d=2$ is rather special, so that we still find it reasonable to conjecture that for $d>2$ no entangled density matrix admits an LCHV model. We can neither prove nor disprove this conjecture. Recently, necessary and sufficient criteria for the quantum state to be entangled have been found [15,16. However, we are unable to use these criteria in our framework. In the following we shall present some partial results.

\section{B. Popescu's result: there is no LCHV model for the Werner states for $d \geq 5$}

A CHV model for a quantum state $\rho$ naturally yields one for collapsed states arising from $\rho$. Consider a measurement of $A$, and suppose the outcome is $a$. The collapsed quantum state is $\rho^{\prime}=\left(P_{a} \rho P_{a}\right) / \operatorname{tr}\left(\rho P_{a}\right)$. From a $\mathrm{CHV}$ model $(\Omega, \mathbb{P}, X)$ for $\rho$ one constructs in the obvious way one for $\rho^{\prime}$ : Let 


$$
\Omega^{\prime}:=\left\{X_{A}=a\right\}, \mathbb{P}^{\prime}=\frac{\left.\mathbb{P}\right|_{\Omega^{\prime}}}{\mathbb{P}\left(\Omega^{\prime}\right)}
$$

and put on $\Omega^{\prime}$

$$
\begin{aligned}
& X_{A^{1}, \ldots, A^{n}}^{\prime}:=X_{A^{1}, \ldots, A^{n}}^{A} \\
& \quad:=\left(X_{A^{1}}^{A}, X_{A^{2}}^{A, A^{1}}, \ldots, X_{A^{n}}^{A, A^{1}, \ldots, A^{n-1}}\right)
\end{aligned}
$$

This model gives the correct quantum probabilities (3) and satisfies the causality condition (四). Moreover,

$$
X_{A^{n}}^{A^{1}, \ldots, A^{n-1}}=X_{A^{n}}^{A, A^{1}, \ldots, A^{n-1}}
$$

Furthermore, if $(\Omega, \mathbb{P}, X)$ is a local CHV model for $\rho$, then $\left(\Omega^{\prime}, \mathbb{P}^{\prime}, X^{\prime}\right)$ is a local CHV model for the collapsed state $\rho^{\prime}$. In our framework, Popescu's result takes the following form: There is no LCHV model for the Werner states $W$ (Eq. (1)) for $d \geq 5$ since there is no such model for the collapsed state $W^{\prime}$ (Eq. (2)) for $d \geq 5$.

We also wish to note the absolutely crucial role played by causality here: Regardless of whether or not it is local, if $(\Omega, \mathbb{P}, X)$ were not causal then $\left(\Omega^{\prime}, \mathbb{P}^{\prime}, X^{\prime}\right)$ need not be well defined, since $\Omega^{\prime}$ could then depend upon the later measurements.

\section{A counterexample in $d=2$ and "almost counterexamples" in $d \geq 3$}

A possible way to come to grips with our conjecture is to find a counterexample. We shall now discuss a family of generalized Werner states (compare Eq. (11))

$$
W=\frac{1}{d}\left(\frac{1}{d}+c\right) I-c F
$$

which may turn out to provide counterexamples. If the real parameter $c$ varies between 0 and $\frac{1}{d^{2}-d}$ these states are normalized $(\operatorname{tr} W=1)$ and positive. Werner has shown that the states (9) are entangled if and only if $\operatorname{tr}(F W)<0$, which translates to $c>\frac{1}{d\left(d^{2}-1\right)}$. For $c=\frac{1}{d^{2}}$ we recover the original Werner states, for which Werner has constructed a stochastic LHV1 model. Since for $c \leq \frac{1}{d^{2}}, W$ is a mixture of the original Werner state and a multiple of the identity, which clearly allows for an LHV1 model, by the mixing construction of section ПIIA all $W$ with $c \leq \frac{1}{d^{2}}$ allow for an LHV1 model.

For the $d=2$ Werner state, Popescu has already remarked that his nonlocality argument does not apply [9]. We will now in fact construct an LCHV model for the state $W$ with $c \leq \frac{1}{4}$ with the help of the LHV1 model just described. In a two-dimensional Hilbert space any nontrivial, i.e., $\neq$ const.I, observable is nondegenerate, so that its projectors are onto onedimensional subspaces. Therefore after the measurement of the first two local observables $A^{1}$ and $B^{1}$ the collapsed state is a pure product state,

\footnotetext{
${ }^{5}$ We may assume without loss of generality that nontrivial measurements are performed on each subsystem.
} 


$$
\begin{aligned}
W^{\prime} & =\frac{\left(P_{A^{1}=a}^{(1)} \otimes P_{B^{1}=b}^{(2)}\right) W\left(P_{A^{1}=a}^{(1)} \otimes P_{B^{1}=b}^{(2)}\right)}{\operatorname{tr}\left(P_{A^{1}=a}^{(1)} \otimes P_{B^{1}=b}^{(2)}\right) W} \\
& =P_{A^{1}=a}^{(1)} \otimes P_{B^{1}=b}^{(2)},
\end{aligned}
$$

and hence has an LCHV model. Moreover, the states $P_{A^{1}=a}^{(1)}$ and $P_{B^{1}=b}^{(2)}$ describing the subsystems after the measurements do not depend on what measurement has been performed on the other subsystem. Therefore given an LHV1 model $\left(\Omega^{(W)}, \mathbb{P}^{(W)}, X^{(W)}\right)$ for the state $W$ and, for all pure states $\alpha=P_{A^{1}=a}^{(1)}$ and $\beta=P_{B^{1}=b}^{(2)}$, CHV models $\left(\Omega^{(1)}, \mathbb{P}^{(1)}, X^{(1, \alpha)}\right)$ and $\left(\Omega^{(2)}, \mathbb{P}^{(2)}, X^{(2, \beta)}\right)$ (where, without loss of generality, we have assumed that the probability spaces $\left(\Omega^{(1)}, \mathbb{P}^{(1)}\right)$ and $\left(\Omega^{(2)}, \mathbb{P}^{(2)}\right)$ don't depend on $\alpha$ and $\beta$, respectively) one can obtain an LCHV model by a simple coupling of the LHV1 model with the CHV models:

Let

$$
\Omega:=\Omega^{(W)} \times \Omega^{(1)} \times \Omega^{(2)}, \quad \mathbb{P}:=\mathbb{P}^{(W)} \times \mathbb{P}^{(1)} \times \mathbb{P}^{(2)}
$$

and

$$
\begin{aligned}
X_{A^{1}, \ldots, A^{n}}(\omega) & :=\left(X_{A^{1}}^{(W)}\left(\omega^{(W)}\right), X_{A^{2}, \ldots, A^{n}}^{A^{1}}\left(\omega^{(W)}, \omega^{(1)}\right)\right), \\
X_{B^{1}, \ldots, B^{m}}(\omega) & :=\left(X_{B^{1}}^{(W)}\left(\omega^{(W)}\right), X_{B^{2}, \ldots, B^{m}}^{B^{1}}\left(\omega^{(W)}, \omega^{(2)}\right)\right),
\end{aligned}
$$

with

$$
X_{A^{2}, \ldots, A^{n}}^{A^{1}}\left(\omega^{(W)}, \omega^{(1)}\right):=X_{A^{2(1)}, \ldots, A^{n(1)}}^{\left(1, \alpha_{A^{1}}\left(\omega^{(W)}\right)\right.}\left(\omega^{(1)}\right)
$$

where

$$
\alpha_{A^{1}}\left(\omega^{(W)}\right)=P_{A^{1}=X_{A^{1}}^{(W)}(\omega(W)}^{(1)},
$$

and

$$
X_{B^{2}, \ldots, B^{m}}^{B^{1}}\left(\omega^{(W)}, \omega^{(2)}\right):=X_{B^{2(2)}, \ldots, B^{m(2)}}^{\left(2, \beta_{B^{1}}\left(\omega^{(W)}\right)\right.}\left(\omega^{(2)}\right)
$$

where

$$
\beta_{B^{1}}\left(\omega^{(W)}\right)=P_{B^{1}=X_{B^{1}}^{(W)}(\omega(W)}^{(2)} \cdot
$$

It is easy to see that $(\Omega, \mathbb{P}, X)$ defines an LCHV model for $W$. Thus we have found a family of entangled states, namely the generalized Werner states (9) with $\frac{1}{6}<c \leq \frac{1}{4}$ in a $2 \times 2$-dimensional Hilbert space, which nevertheless admit an LCHV model. We shall call these states $W_{d=2}$ states.

We shall next show that in dimension $d \geq 3$ the (entangled) Werner states with $c \in$ $\left(\frac{1}{d\left(d^{2}-1\right)}, \frac{1}{d\left(d^{2}-1\right)-d^{2}}\right]$ become non-entangled already after one nontrivial local measurement (on one side). (This is trivially true in $d=2$.) We shall later refer to these states as $W_{d \geq 3}$ states. To show that they are left in a non-entangled state after one nontrivial local measurement, note that if $P$ is a projector on a $(d-1)$-dimensional subspace of $\mathbb{C}^{d}$, the 
collapsed state $W^{\prime}=(P \otimes P) W(P \otimes P) / \operatorname{tr}(W(P \otimes P))$ on the subspace $\operatorname{Ran} P \otimes \operatorname{Ran} P$ has again the form (9) with

$$
c^{\prime}=\frac{c d^{2}}{(d-1)(d-c d-1)}
$$

For $c^{\prime} \leq \frac{1}{(d-1)\left((d-1)^{2}-1\right)} \Leftrightarrow c \leq \frac{1}{d\left(d^{2}-1\right)-d^{2}}$ the state $W^{\prime}$ is non-entangled, and from this it follows that the same thing is true when the range of $P$ has dimension $<(d-1)$. Thus we calculate

$$
\begin{aligned}
(P \otimes & I) W(P \otimes I) \\
= & \left(P \otimes\left(P+P^{\perp}\right)\right) W\left(P \otimes\left(P+P^{\perp}\right)\right) \\
= & (P \otimes P) W(P \otimes P) \\
& +\frac{1}{d}\left(\frac{1}{d}+c\right)\left(P \otimes P^{\perp}\right) I\left(P \otimes P^{\perp}\right) \\
& -c\left(P \otimes P^{\perp}\right) F\left(P \otimes P^{\perp}\right) \\
& -c\left(P \otimes P^{\perp}\right) F(P \otimes P) \\
& -c(P \otimes P) F\left(P \otimes P^{\perp}\right) .
\end{aligned}
$$

Here $P^{\perp}=I-P$. The first term in the sum (10) is non-entangled due to the foregoing argument, the second term is obviously non-entangled, and a straightforward calculation shows that the last three terms vanish. Thus already after one local measurement the collapsed state is non-entangled and therefore local.

Thus for any $d \geq 2$ we have found states that are entangled, admit an LHV1 model, and are left after any local measurement in a non-entangled state, where there is of course an LCHV model for further measurements. However, we cannot extend our construction of an LCHV model for the original state from $d=2$ to $d \geq 3$, since the range of the projectors may have dimension $>1$, in which case the state after one local measurement on each side may depend irreducibly on both measurements, i.e., in such a manner that random variables on one side must have some dependence on what observable was first measured on the other side: The LHV1 model for the state itself contains random variables $X_{A^{1}}$ and $X_{B^{1}}$ for the first measurements. The existence of an LCHV model for all collapsed states yields the follow-up random variables $X_{A^{2}, \ldots, A^{n}}^{A^{1}, B^{1}}$ and $X_{B^{2}, \ldots, B^{m}}^{A^{1}, B^{1}}$, not the follow-up random variables $X_{A^{2}, \ldots, A^{n}}^{A^{1}}$ and $X_{B^{2}, \ldots, B^{m}}^{B^{1}}$ required for an LCHV model for the original state. Nevertheless, it does follow that it is impossible to reveal the nonlocality of these states by a Popescu-type argument, namely by producing a nonlocal state violating a Bell inequality by local measurements.

\section{A Nonlocality Classification Scheme}

We have shown that for $d=2$ the main conjecture is wrong, i.e. there are entangled mixed states allowing for an LCHV model. For the $W_{d \geq 3}$ entangled mixed states allowing for an LHV1 model for the first measurement and an LCHV model for all further measurements, both possibilities, either the existence of an LCHV model or its nonexistence, are very interesting. 
In the first case the main conjecture is indeed wrong: entangledness is not equivalent to the nonexistence of an LCHV model. If, on the other hand, it turns out that there is no LCHV model for these states, a new nonlocality complexity class emerges: a class of entangled mixed states with nonlocality that is more deeply hidden than that of the Popescu/Werner example.

We are thus lead to a nonlocality classification scheme which we shall briefly describe. We classify the nonlocality of quantum states by means of a pair $(N, n)$ of positive integers, the indices of nonlocality. Larger values of these indices correspond to more deeply hidden nonlocality. The first index $N$ corresponds to the length of the sequence of measurements necessary to reveal the nonlocality, and the second index $n$ conveys the degree to which the state can be transformed, by performing local measurements upon the system, to one in which the nonlocality is more manifest. More precisely, the first index denotes the smallest integer $N$ such that there is no LCHVN model (i.e., no local causal model for sequences of maximal length $N$ ) for the states in this class. For example, entangled pure states have $N=1$ while Werner's states for $d \geq 5$ have $N=2$. For states that have an LCHV model we put $N=\infty$.

Suppose a state has finite first index of nonlocality $N$. After one measurement the state collapses into one that clearly has first index at least $N-1$. We say "at least" because it may well be the case that the collapsed state has an LCHV $k$ model for $k \geq N-1$, i.e., has index $N$ or greater. For example, for our $W_{d \geq 3}$ states, the state after one nontrivial measurement has index $N=\infty$. In contrast, in Popescu's example the index $N=2$ can be reduced to $N=1$ by one measurement. One may thus hope to reduce the first index of nonlocality by performing a sequence of measurements. The smallest number to which it can, with nonvanishing probability, be reduced will be denoted by $n$. Note that if there is no LCHV model for a $W_{d \geq 3}$ state, the nonlocality of this state must be irreducible, i.e., we must have that $n=N$.

$N=\infty$ for nonentangled states, which are completely local. But there are also entangled states allowing for an LCHV model, as is the case, for example, with the $W_{d=2}$ states. It would seem natural to conclude that such states do not produce any nonlocal effects. However, a recent result by Bennett et al. [17] shows that this conclusion is wrong. They found a nonlocality argument employing a sequence of local unitary operations and measurements on an ensemble of systems each in such a state - the unitary operations and measurements are performed on states of the form $W \otimes W \otimes \cdots \otimes W$ on the Hilbert space $\mathbb{C}^{4} \otimes \mathbb{C}^{4} \otimes \cdots \otimes \mathbb{C}^{4}$. These states possess nonlocality that is even more deeply hidden than what we have discussed so far. This suggests now as the second classification index $n$ in the case $N=\infty$ the minimal number of copies of the system in the ensemble required for such a nonlocality argument. If there is no finite $n$ with this property, we put $n=\infty$.

The following table contains the known results about this classification:

\footnotetext{
${ }^{6}$ Abstract considerations show that the existence of an LCHVk model for all finite $k$ implies the existence of an LCHV model.
} 


\begin{tabular}{|c|c|}
\hline$(N, n)$ & examples \\
\hline$(1,1)$ & entangled pure states \\
$(2,1)$ & Werner states for $d \geq 5$ \\
$(k, k)$ for some $k \geq 2$ & $W_{d \geq 3}$ states having no LCHV model \\
$(\infty, k)$ with $k<\infty$ & $W_{d=2}$ states \\
$(\infty, ?)$ & $W_{d \geq 3}$ states which have an LCHV model \\
$(\infty, \infty)$ & nonentangled states \\
\hline
\end{tabular}

A natural conjecture - a weakening of our main conjecture - is that the only states in class $(\infty, \infty)$ are the nonentangled ones.

A further possibility to exhibit nonlocality is the use of generalized measurements, which we shall discuss below. It may turn out that analogous classification indices are of interest also for generalized measurements, but we have no results in that direction.

\section{GENERALIZED OBSERVABLES}

\section{A. Hidden variables models for generalized observables}

We shall now extend the allowed class of observables. In standard quantum theory the measurement of an observable is associated with a self-adjoint operator, which corresponds by the spectral theorem to a projection-valued (PV) measure. But the concept of PV measure can be generalized in a natural way to that of positive operator valued (POV) measure. POV's have been employed to construct generalized quantum observables for which the usual framework of self-adjoint operators has been unsuccessful, mainly in the fields of quantum optics and the theory of open quantum systems [18. Moreover, POV's emerge naturally from an analysis of quantum experiments in Bohmian mechanics [19]. A POV measure is a set function $M$, which maps measurable subsets $\Delta \subset \mathbb{R}$ to bounded positive operators $M(\Delta)$, such that for any quantum state $\rho$

$$
\Delta \mapsto \mu_{M}^{\rho}(\Delta):=\operatorname{tr} \rho M(\Delta)
$$

is an ordinary probability measure on $\mathbb{R}$, i.e., $M(\mathbb{R})=I$ and for any sequence of disjoint measurable sets $\left(\Delta_{i}\right)_{i \in \mathbb{N}}, \Delta_{i} \subset \mathbb{R}$,

$$
M\left(\bigcup_{i \in \mathbb{N}} \Delta_{i}\right)=\sum_{i \in \mathbb{N}} M\left(\Delta_{i}\right)
$$

$\mu_{M}^{\rho}(\Delta)$ is the probability in the quantum state $\rho$ for the outcome of the measurement associated with the POV $M$ to be in $\Delta$. The complete formal description of a measurement of a generalized observable, whose outcome statistics are given by a $\mathrm{POV} M$, is provided by the so-called operations $R_{\delta}$, a family of bounded linear operators, describing the change of quantum state during the measurement if the outcome $\delta \in \mathbb{R}$ is obtained:

$$
\rho \rightarrow \tilde{\rho}=\frac{R_{\delta} \rho R_{\delta}^{\dagger}}{\operatorname{tr} \rho R_{\delta}^{\dagger} R_{\delta}} .
$$

The operations $R_{\delta}$ determine the POV $M$ via 


$$
M(\Delta)=\sum_{\delta \in \Delta} R_{\delta}^{\dagger} R_{\delta}
$$

POV measures include as a special case the PV measures where all the positive operators are orthogonal projections. (In an "ideal" measurement of a PV $M$ the operations $R_{\delta}$ are equal to the projections $P_{\delta}=M(\delta):=M(\{\delta\})$ corresponding to the usual collapse rule. However, in general there are huge classes of possible operations $R_{\delta}$ giving rise to the same POV or PV via $M(\delta)=R_{\delta}^{\dagger} R_{\delta}$.)

As with ordinary observables, we consider here only generalized observables having a discrete set of possible values $\delta$, and we denote by $\mathcal{R}$ the corresponding family of operations $\left\{R_{\delta}\right\}$.

Our definitions of the various kinds of hidden variables models may without effort be generalized to cover measurements of generalized observables: just consider, instead of sequences of observables $O^{1}\left(t_{1}\right), \ldots, O^{n}\left(t_{n}\right)$, sequences $\mathcal{R}^{1}\left(t_{1}\right), \ldots, \mathcal{R}^{n}\left(t_{n}\right) . \sigma\left(\mathcal{R}^{k}\right)$ is the generalized spectrum of the generalized measurement $\mathcal{R}^{k}$, i.e., the set of possible outcomes $\left\{\delta \mid R_{\delta}^{k} \neq 0\right\}$. Eq. (3) for the quantum mechanical probabilities gets replaced by

$$
\begin{aligned}
& \mathbb{P}\left(X_{\mathcal{R}^{1}\left(t_{1}\right), \ldots, \mathcal{R}^{n}\left(t_{n}\right)}=\left(\delta_{1}, \ldots, \delta_{n}\right)\right) \\
& \quad=\operatorname{tr}\left(R_{\delta_{n}}^{n}\left(t_{n}\right) \cdots R_{\delta_{1}}^{1}\left(t_{1}\right) \rho R_{\delta_{1}}^{1 \dagger}\left(t_{1}\right) \cdots R_{\delta_{n}}^{n \dagger}\left(t_{n}\right)\right) .
\end{aligned}
$$

The causality and locality condition do not change at all, and similarly the definition of a stochastic local causal hidden variables model may be trivially extended to cover measurements of generalized observables. We shall denote models which apply to generalized measurements by a $\mathrm{G}$ at the end of their abbreviation.

The equivalence theorem mentioned in Section [1], as well as its proof in Appendix A, holds just as well for models for generalized observables, as do the results in Sections IIIA and IIIB. However, our $d=2$ counterexample in Section III to the conjecture that only non-entangled states admit LCHV models does not generalize to LCHVG models, since Werner's model is only for standard observables, as well as because the state resulting from the first local measurements of generalized observables on the two sides may depend irreducibly on both measurements, just as discussed in the second to the last paragraph of Section IIIQ. Thus the conjecture for generalized observables, i.e., the conjecture that only non-entangled states admit LCHVG models, may be true in all dimensions $d \geq 2$.

However, in this context it would appear natural to first analyze a simpler conjecture which has been raised by Popescu [9]: Is the existence of an LHV1G model equivalent to the state being non-entangled? Is it possible to reveal the nonlocality of any entangled state by considering measurements at only a single time, at least if generalized observables are taken into account? Here again the Werner states serve as the first check: can Werner's model be extended to cover also generalized measurements? A positive answer to this question would answer Popescu's conjecture to the negative. In the following section we provide a partial positive answer.

\footnotetext{
${ }^{7}$ Actually only the first two measurements (one on each side) have to be ideal measurements; the following ones may also be generalized measurements.
} 


\section{B. Extension of LHV1 models for standard observables to certain generalized observables}

The LHV1 model constructed by Werner [8] for the states (11) covers only measurements of ordinary observables. In this section we will show that any LHV1 model for ideal measurements can be extended to a model for certain special generalized observables, namely those given by a POV involving only commuting operators $M(\delta)$, p which we shall call commuting POV's. This covers for example all two-valued POV's: If $M\left(\delta_{1}\right)+M\left(\delta_{2}\right)=I$, then $M\left(\delta_{1}\right)=I-M\left(\delta_{2}\right)$ commutes with $M\left(\delta_{2}\right)$. Moreover, many of the standard examples of POV's are commuting POV's. For example, given an observable $O=\sum_{i} o_{i} P_{o_{i}}$ in its spectral representation , we may consider the "smeared-out" projections

$$
M\left(o_{i}\right)=\sum_{j} t_{i j} P_{o_{j}}
$$

with $t_{i j} \geq 0$ and $\sum_{i} t_{i j}=1$, clearly a commuting POV. Here $t_{i j}$ is the probability of obtaining the result $o_{i}$ when the system is in an eigenstate corresponding to the eigenvalue $o_{j}$. Also, the model of Gisin [10] for a filtering process is described by a commuting POV.

We will now construct from an LHV1 model on $(\Omega, \mathbb{P})$ for ordinary observables a stochastic LHV1 model, also on $(\Omega, \mathbb{P})$, for all generalized observables governed by commuting POV's. By the equivalence result of Appendix A we have simultaneously a deterministic LHV1 model for these generalized observables. Thus generalized observables described by commuting POV's cannot reveal the "hidden nonlocality" of the Werner states with measurements at a single time.

Let $M(\alpha)=M^{(1)}(\alpha) \otimes I$ and $\widetilde{M}(\beta)=I \otimes \widetilde{M}^{(2)}(\beta)$ each be commuting POV's on a finite dimensional Hilbert space. (Using the notion of spectral measures, the following calculation can also be done for Hilbert spaces of infinite dimension [14.) A joint measurement of the corresponding generalized observables is described by the $\operatorname{POV} M(\alpha) \widetilde{M}(\beta)$, and the quantum mechanical probability for obtaining outcomes $\alpha$ and $\beta$ in this measurement in the state $\rho$ is thus

$$
\operatorname{tr} \rho\left(M^{(1)}(\alpha) \otimes \widetilde{M}^{(2)}(\beta)\right)
$$

These are the probabilities that must be recovered by a stochastic local hidden variables model.

The positive commuting operators $M^{(1)}(\alpha)$ have a joint spectral representation $M^{(1)}(\alpha)=\sum_{j} m_{\alpha}^{j} P_{j}^{(1)}$ with $0 \leq m_{\alpha}^{j} \leq 1$, where all the $P_{j}^{(1)}$ are projections onto onedimensional orthogonal subspaces and are independent of $\alpha$. Similarly, $\widetilde{M}^{(2)}(\beta)=\sum_{k} \widetilde{m}_{\beta}^{k} \widetilde{P}_{k}^{(2)}$ with $0 \leq \widetilde{m}_{\beta}^{k} \leq 1$.

Let $A=\sum_{j} j P_{j}^{(1)}$ and $B=\sum_{k} k \widetilde{P}_{k}^{(2)}$. Since we have assumed the existence of an LHV1 model $(\Omega, \mathbb{P}, X)$, we have random variables $X_{A}, X_{B}$ for which

\footnotetext{
${ }^{8}$ Since LHV1 models concern only a single time, the operations $R_{\delta}$ describing the change of state during measurement are not relevant here.
} 


$$
\mathbb{P}\left(X_{A}=j, X_{B}=k\right)=\operatorname{tr} \rho\left(P_{j}^{(1)} \otimes P_{k}^{(2)}\right) .
$$

We define

$$
Q_{M}(\alpha \mid \omega):=\sum_{j} m_{\alpha}^{j} \mathbb{1}_{\{j\}}\left(X_{A}(\omega)\right)
$$

and

$$
Q_{\widetilde{M}}(\beta \mid \omega):=\sum_{k} \widetilde{m}_{\beta}^{k} \mathbb{1}_{\{k\}}\left(X_{B}(\omega)\right)
$$

Then

$$
\begin{aligned}
\int_{\Omega} & Q_{M}(\alpha \mid \omega) Q_{\widetilde{M}}(\beta \mid \omega) d \mathbb{P}(\omega) \\
& =\sum_{j, k} m_{\alpha}^{j} \widetilde{m}_{\beta}^{k} \int_{\Omega} \mathbb{1}_{\{j\}}\left(X_{A}(\omega)\right) \mathbb{1}_{\{k\}}\left(X_{B}(\omega)\right) d \mathbb{P}(\omega) \\
& =\sum_{j, k} m_{\alpha}^{j} \widetilde{m}_{\beta}^{k} \mathbb{P}\left(X_{A}=j, X_{B}=k\right) \\
& =\sum_{j, k} m_{\alpha}^{j} \widetilde{m}_{\beta}^{k} \operatorname{tr} \rho\left(P_{j}^{(1)} \otimes P_{k}^{(2)}\right) \\
& =\operatorname{tr} \rho\left(\sum_{j} m_{\alpha}^{j} P_{j}^{(1)} \otimes \sum_{k} \widetilde{m}_{\beta}^{k} \widetilde{P}_{k}^{(2)}\right) \\
& =\operatorname{tr} \rho M(\alpha) \widetilde{M}(\beta)
\end{aligned}
$$

Moreover, since

$$
I^{(1)}=\sum_{\alpha} M(\alpha)=\sum_{j} \sum_{\alpha} m_{\alpha}^{j} P_{j}^{(1)}
$$

it follows that

$$
\sum_{\alpha} m_{\alpha}^{j}=1
$$

for all $j$. Therefore the probability distribution $Q_{M}(. \mid \omega)$ is properly normalized:

$$
\sum_{\alpha} Q_{M}(\alpha \mid \omega)=1
$$

and analogously for $Q_{\widetilde{M}}(. \mid \omega)$. Thus from an LHV1 model on $(\Omega, \mathbb{P})$ for ordinary observables we have constructed a stochastic LHV1 model, also on $(\Omega, \mathbb{P})$, for all generalized observables governed by commuting POV's.円

\footnotetext{
${ }^{9}$ Notice that the simpler definition $Q_{M}(\alpha \mid \omega)=X_{M(\alpha)}(\omega), Q_{\widetilde{M}}(\beta \mid \omega)=X_{\widetilde{M}(\beta)}(\omega)$ also yields the quantum mechanical probabilities (12), and it does so regardless of whether or not $M$ and $\widetilde{M}$ are each commuting POV's. Note however that the normalization $\sum_{\alpha} Q_{M}(\alpha \mid \omega)=1$ need not hold with this definition, since it does not follow from $\sum_{\alpha} M(\alpha)=I$ that $\sum_{\alpha} X_{M(\alpha)}=1$, regardless of whether the $M(\alpha)$ fail to commute (recall von Neumann's no-hidden-variables theorem [1, page 4]) or do indeed commute (recall Gleason's theorem [1, page 6]). Note also (again recalling Gleason's theorem) that $X_{M(\alpha)}=X_{m_{\alpha}(A)}$ need not agree with $m_{\alpha}^{X_{A}}$ even in our commuting case.
} 
In this connection, it is interesting to comment on a recent work of Gisin [10], who in Section 3 of his paper gives an example of an apparent violation of the Bell-CHSH inequality even for non-entangled states for generalized observables described by commuting POV's. However, his point that a "careless application of generalized quantum measurements can violate Bell's inequality even for mixtures of product states" is misleading: the observed violation of the Bell-CHSH inequality is not in fact due to the application of gen-

eralized quantum measurements, but rather to a misapplication of the inequality itself- to conditional expectations in which the conditioning depends upon the measurements under consideration [20].

\section{CONCLUSION}

We have analyzed some questions concerning the classification of local states in quantum theory. There are two intuitive notions of "locality" for quantum states: (i) the state is nonentangled, i.e., a product state or a mixture of product states, and (ii) the state admits a local and causal hidden variables model.

For pure quantum states it is well-known that the non-entangledness is equivalent to the existence of an LHV1 model. We have shown that this equivalence extends to LCHVG models. For mixed states, the equivalence between non-entangledness and the existence of an LCHV model (for $d>2$ ) is our main conjecture. We have shown that (i) $\Rightarrow$ (ii), and have verified that pure states and Werner states with $d \geq 5$ conform to our conjecture. Furthermore, we have described counterexamples in dimension $d=2$, namely the states $W_{d=2}$, and a class of "almost-counterexamples" in higher dimensions, the states $W_{d \geq 3}$. These "almost-counterexamples" leave two possibilities: Either the main conjecture is wrong in any dimension, or there is a more deeply hidden "irreducible nonlocality," in the sense explained in Subsection IIID. In connection with the latter possibility we have proposed two new indices of nonlocality as the basis of a nonlocality complexity classification.

Another conjecture concerning the classification of local states, which has been raised by Popescu [9], is that non-entangled states are the only states admitting LHV1G models for all possible measurements, including those described by POV's. From our result in Section IV it follows that to exclude the possibility of an LHV1 model for the entangled Werner states (or for any other states admitting an LHV1 model) POV's that are close to PV's in the sense that they contain only commuting operators will not help.

Furthermore, we have extended Fine's result on the equivalence between the existence of deterministic and stochastic LHV1 models to LCHVG models. Besides the usefulness of this result for the construction of models, we regard it as further evidence that our notion of a local causal hidden variables model adequately captures the relevant physical ideas.

\section{ACKNOWLEDGMENTS}

We thank the referee for valuable comments and suggestions. This work was supported in part by the DFG, by NSF Grant No. DMS-9504556, and by the INFN. 


\section{APPENDIX A: EQUIVALENCE BETWEEN THE EXISTENCE OF DETERMINISTIC AND STOCHASTIC HIDDEN VARIABLES MODELS}

A (deterministic) LCHV model $(\Omega, \mathbb{P}, X)$ forms a degenerate stochastic LCHV model if one defines $\widetilde{\Omega}=\Omega, \widetilde{\mathbb{P}}=\mathbb{P}$, and sets $Q_{O^{1}\left(t_{1}\right), \ldots, O^{n}\left(t_{n}\right)}\left(o_{1}, \ldots, o_{n} \mid \widetilde{\omega}\right):=1$ if $X_{O^{1}\left(t_{1}\right), \ldots, O^{n}\left(t_{n}\right)}(\widetilde{\omega})=$ $\left(o_{1}, \ldots, o_{n}\right)$ and 0 otherwise. Conversely, from a given stochastic model one can construct a (deterministic) LCHV model as follows: Let $\rho$ be a quantum state with a stochastic LCHV model $(\widetilde{\Omega}, \widetilde{\mathbb{P}}, Q)$. For all $n \in \mathbb{N}$, all sequences of local observables $A^{k}$ for the first subsystem and all $a_{k} \in \sigma\left(A^{k}\right), k=1 \ldots n$, and all $\widetilde{\omega} \in \widetilde{\Omega}$, there exist independent random variables

$$
\widehat{X}_{A^{n}, a_{1}, \ldots, a_{n-1}, \widetilde{\omega}}^{A^{1}, \ldots, A^{n-1}}: \Omega_{A} \rightarrow \sigma\left(A^{n}\right)
$$

with distribution $\mathbb{P}_{A}\left(\widehat{X}_{A^{n}, a_{1}, \ldots, a_{n-1}, \widetilde{\omega}}^{A^{1}, \ldots, A^{n-1}}=a_{n}\right)=Q_{A^{1}, \ldots, A^{n}}\left(a_{n} \mid a_{1}, \ldots, a_{n-1}, \widetilde{\omega}\right)$ on some probability space $\left(\Omega_{A}, \mathbb{P}_{A}\right)$. (The canonical choice is the huge product space $\prod_{\alpha} \mathbb{R}$ over all possible such choices $\alpha$, equipped with the product measure $\prod_{\alpha} \mu_{\alpha}$ with $\mu_{\alpha}$ the conditional probability distribution $Q_{A^{1}, \ldots, A^{n}}\left(a_{n} \mid a_{1}, \ldots, a_{n-1}, \widetilde{\omega}\right)$ corresponding to $\alpha$, and with the random variables given by the corresponding projections.) Similarly, for all $m \in \mathbb{N}$, all sequences of local observables $B^{k}$ for the second subsystem and all $b_{k} \in \sigma\left(B^{k}\right), k=1 \ldots m$, and all $\widetilde{\omega} \in \widetilde{\Omega}$ define independent random variables

$$
\widehat{X}_{B^{m}, b_{1}, \ldots, b_{m-1}, \widetilde{\omega}}^{B^{1}, \ldots, B^{m-1}}: \Omega_{B} \rightarrow \sigma\left(B^{m}\right)
$$

on some probability space $\left(\Omega_{B}, \mathbb{P}_{B}\right)$ with the distribution $\mathbb{P}_{B}\left(\widehat{X}_{B^{m}, b_{1}, \ldots, b_{m-1}, \widetilde{\omega}}^{B^{1}, \ldots B^{m-1}}=b_{m}\right)=$ $Q_{B^{1}, \ldots, B^{m}}\left(b_{m} \mid b_{1}, \ldots, b_{m-1}, \widetilde{\omega}\right)$. Now put

$$
\Omega=\widetilde{\Omega} \times \Omega_{A} \times \Omega_{B}, \quad \mathbb{P}=\widetilde{\mathbb{P}} \times \mathbb{P}_{A} \times \mathbb{P}_{B}
$$

and define inductively all $X_{A^{1}, \ldots, A^{n}}$ and $X_{B^{1}, \ldots, B^{m}}$ using (5): for $\omega=\left(\widetilde{\omega}, \omega_{A}, \omega_{B}\right)$

$$
\begin{aligned}
& X_{A^{1}}(\omega)=\widehat{X}_{A^{1}, \widetilde{\omega}}\left(\omega_{A}\right), \quad X_{B^{1}}(\omega)=\widehat{X}_{B^{1}, \widetilde{\omega}}\left(\omega_{B}\right), \\
& X_{A^{n}}^{A^{1}, \ldots, A^{n-1}}(\omega)=\widehat{X}_{A^{n}, X_{A^{1}, \ldots, A^{n-1}}(\omega), \widetilde{\omega}}^{A^{1}, \ldots, A^{n-1}}\left(\omega_{A}\right), \\
& X_{B^{m}}^{B^{1}, \ldots, B^{m-1}}(\omega)=\widehat{X}_{B^{m}, X_{B^{1}, \ldots, B^{m-1}}^{B^{1}}(\omega), \widetilde{\omega}}\left(\omega_{B}\right) .
\end{aligned}
$$

One easily sees that this defines an LCHV model for the quantum state $\rho$. (For example,

$$
\begin{aligned}
\mathbb{P} & \left(X_{A^{1}, A^{2}}=\left(a_{1}, a_{2}\right)\right) \\
& =\int_{\widetilde{\Omega}} d \widetilde{\mathbb{P}}(\widetilde{\omega}) \int_{\Omega_{A}} d \mathbb{P}_{A}\left(\omega_{A}\right) \int_{\Omega_{B}} d \mathbb{P}_{B}\left(\omega_{B}\right) \mathbb{1}_{\left\{X_{A^{1}}=a_{1}, X_{A^{2}}^{A^{1}}=a_{2}\right\}} \\
& =\int_{\widetilde{\Omega}} d \widetilde{\mathbb{P}}(\widetilde{\omega}) \int_{\Omega_{A}} d \mathbb{P}_{A}\left(\omega_{A}\right) \int_{\Omega_{B}} d \mathbb{P}_{B}\left(\omega_{B}\right) \\
& =\int_{\widetilde{\Omega}} d \widetilde{\mathbb{P}}(\widetilde{\omega}) \mathbb{P}_{A}\left(\widehat{X}_{A^{1}, \widetilde{\omega}^{1}, \widetilde{\omega}}=a_{1}, \widehat{X}_{A^{2}, a_{1}, \widetilde{\omega}}^{A^{1}}=a_{1}, \widehat{X}_{A^{2}, a_{1}, \sim^{1}}\left(\omega_{A}\right)=a_{2}\right\}
\end{aligned}
$$




$$
\begin{aligned}
& =\int_{\widetilde{\Omega}} d \widetilde{\mathbb{P}}(\widetilde{\omega}) \mathbb{P}_{A}\left(\widehat{X}_{A^{1}, \widetilde{\omega}}=a_{1}\right) \mathbb{P}_{A}\left(\widehat{X}_{A^{2}, a_{1}, \widetilde{\omega}}^{A^{1}}=a_{2}\right) \\
& =\int_{\widetilde{\Omega}} d \widetilde{\mathbb{P}}(\widetilde{\omega}) Q_{A^{1}}\left(a_{1} \mid \widetilde{\omega}\right) Q_{A^{1}, A^{2}}\left(a_{2} \mid a_{1}, \widetilde{\omega}\right) \\
& =\int_{\widetilde{\Omega}} d \widetilde{\mathbb{P}}(\widetilde{\omega}) Q_{A^{1}, A^{2}}\left(a_{1}, a_{2} \mid \widetilde{\omega}\right),
\end{aligned}
$$

where the independence of the random variables $\widehat{X}$ is used for the 4 th equation.) 


\section{REFERENCES}

[1] J.S. Bell, Speakable and Unspeakable in Quantum Mechanics (Cambridge University Press, Cambridge, 1987).

[2] A. Einstein, B. Podolsky, and N. Rosen, Phys. Rev. 47, 777 (1935).

[3] A. Aspect, P. Grangier, and G. Roger, Phys. Rev. Lett. 49, 1804 (1982).

[4] D. Bohm, Quantum Theory (Prentice-Hall, Englewood Cliffs, 1951).

[5] A. Fine, Phys. Rev. Lett. 48, 291 (1982).

[6] N. Gisin, Phys. Lett. A 145, 201 (1991).

[7] S. Popescu and D. Rohrlich, Phys. Lett. A 166, 293 (1992).

[8] R.F. Werner, Phys. Rev. A 40, 4277 (1989).

[9] S. Popescu, Phys. Rev. Lett. 74, 2619 (1995).

[10] N. Gisin, Phys. Lett. A 210, 151 (1996).

[11] N.D. Mermin, Notes for a lecture given in Bielefeld, July 1995 (unpublished).

[12] M. Zukowski, R. Horodecki, M. Horodecki, and P. Horodecki, Generalized measurements and local realism, preprint (quant-ph/9608035).

[13] M. Daumer, D. Dürr, S. Goldstein, and N. Zanghì, in Erkenntnis, Proceedings of the International Conference on Probability, Dynamics and Causality, Luino, Italy, 1995, edited by D. Costantini and M.C. Gallavotti (to appear) (quant-ph/9601013).

[14] S. Teufel, Diplomarbeit, Universität München, 1996.

[15] A. Peres, Phys. Rev. Lett. 77, 1413 (1996).

[16] M. Horodecki, P. Horodecki, and R. Horodecki, Phys. Lett. A 223, 1 (1996).

[17] C.H. Bennett, G. Brassard, S. Popescu, B. Schumacher, J. Smolin, and W.K. Wootters, Phys. Rev. Lett. 76, 722 (1996).

[18] E.B. Davies, Quantum Theory of Open Systems (Academic Press, 1976).

[19] M. Daumer, D. Dürr, S. Goldstein, and N. Zanghì, Bohmian mechanics and the role of operators as observables in quantum theory, in preparation.

[20] K. Berndl and S. Teufel, Phys. Lett. A 224, 314 (1997). 